\title{
Pluripotent Stem Cell Models of Early Mammalian Development
}

Peter Baillie-Benson ${ }^{1}$, Naomi Moris ${ }^{1} \&$ Alfonso Martinez Arias ${ }^{1,}{ }^{*}$.

1. Department of Genetics, University of Cambridge, Downing Street, Cambridge, UK CB2 3EH.

* To whom correspondence should be addressed; email: ama11@hermes.cam.ac.uk

\begin{abstract}
Pluripotent stem cells derived from the early mammalian embryo offer a convenient model system for studying cell fate decisions in embryogenesis. The last ten years has seen a boom in the popularity of two-dimensional micropatterns and three-dimensional stem cell culture systems as a way to recreate the architecture and interactions of particular cell populations during development. These methods enable the controlled exploration of cellular organisation and patterning during development, using cell lines instead of embryos. They have established a new class of in vitro model system for pre- and peri-implantation embryogenesis, ranging from models of the blastocyst stage, through gastrulation and towards early organogenesis. This review aims to set these systems in context and to highlight the strengths and suitability of each approach in modelling early mammalian development.
\end{abstract}

\section{Introduction}

Animal development is defined by the assemblage of cells into organs and tissues and their coordinated association into a functionally integrated whole. Our understanding of these processes has largely relied on two complementary approaches. On the one hand Genetics, the isolation and characterization of mutants in organisms such as C. elegans, Drosophila, and more recently zebrafish has revealed the molecular underpinning of developmental events [1]. Through this molecular understanding, narratives have been built that define mechanisms involved in the generation and patterning of cell diversity. Many have centred on notions like 'morphogens' (gradients of molecules that can lead to patterning cell fate decisions) and 'Gene Regulatory Networks' (the organised interactions between gene products). A second approach, Experimental Embryology, had its heyday in the early $20^{\text {th }}$ century using organisms like chickens and frogs, in which Genetics is challenging. It focuses on the abilities of groups of cells to work together and has yielded notions such as 'organizers' (a group of cells shown to be sufficient to induce neural fates), 'induction' (the ability of one tissue to influence another) and the 'community effect' (when the ability of cells to respond to an induction is dependent on its neighbouring cells) [2]. This approach 
can be used to explain how cellular interactions can structure the whole organism from the level of the major body axes down to individual tissues. Although this approach lacks mechanistic insights it has been an important reference for genetic analysis. In an ideal world one would like to blend the two to provide biological understanding across scales.

Mammalian development presents a challenge for both approaches. The slow reproductive time and, often, large size of mammalian organisms creates logistical problems for high-throughput mutant genetic screens. In addition, mammalian embryos are difficult to access and manipulate due to their internal development and their dependence on maternal interactions. Nevertheless, careful experimentation has pieced together a picture of the molecular and cellular events associated with early embryogenesis, with a particular focus on the mouse. These events highlight adaptations to the embryo-maternal interactions necessary for post-implantation development.

Mammalian development begins at fertilization, after which the zygote divides and segregates two lineages that will not primarily contribute to the embryo but create an interface with the mother: the Trophectoderm (TE) generates the placenta and the Primitive Endoderm (PrEnd) the yolk sac [3]. The embryo itself, specified after these lineages, is derived from the epiblast; a cellular ensemble that is asymmetrically encased between the TE and the PrEnd. The epiblast then undergoes gastrulation, a key developmental process that establishes the organised differentiation of the three germ layers (see Figure 1) [4]. Genetic analysis has shown that a sequence of interactions between the embryonic and extra-embryonic lineages creates an axial reference for this process. The first sign of gastrulation is the expression of the T-box transcription factor Brachyury (Bra) at the prospective posterior end of the embryo, and an Epithelial to Mesenchymal Transition (EMT) that is propagated along the midline from posterior to anterior in the form of a dynamic groove, the Primitive Streak. This leads to the specification of the endoderm and the mesoderm. The body plan is laid out by the end of gastrulation with reference to three orthogonal axes: an anteroposterior (AP) axis defined by the head and the brain and one end and the primordium for the thorax at the other; a dorsoventral (DV) axis with the nervous system dorsally and the endoderm ventrally; and the primordia for different mesodermal derivatives organised with respect to a midline that defines a bilateral axis of symmetry (Figure 1) [5].

Embryonic Stem Cells (ESCs), clonal derivatives from early mammalian embryos that can be propagated and differentiated in culture, have become an invaluable tool for the study of 
mammalian development. In particular, the ability to direct their differentiation in culture has provided significant insights into how signals and transcription factor networks interact during cell fate decisions. Importantly, non-adherent cultures have revealed an intrinsic self-organizing ability that has been used to develop three-dimensional mimics of embryonic and fetal organs (organoids). These have expanded our understanding of tissue morphogenesis and created an interesting platform for regenerative medicine [6]. This self-organizing ability of ESCs has also been used to explore the early stages of development in what we could generically call "embryonic organoids" (see Box 1). Here we focus on examples of embryonic organoids and discuss how different systems that have emerged from pluripotent stem cell cultures can be used to model different features of embryogenesis and what we are learning from them.

\section{Embryonic Stem Cells and Micropatterns - models of germ layer organisation}

The embryo is a combination of different cell types in specific proportions that interact to increase complexity over time in creating tissues and organs. However, when ESCs are subject to directed differentiation in culture, they display heterogeneities in gene expression that are dependent on the signalling environment. Heterogeneity in gene expression, particularly of members of the core pluripotency network, produces a dynamic mix of cell states in an ESC culture, with local differences in the spatial organisation and movement of the cells. These heterogeneities are transferred to differentiation choices $[7,8]$ and have provoked discussions of whether such heterogeneities exist in the embryo and play a role in fate assignments and pattern formation, even at the earliest stages of development [9]. The question remains open but the answer needs to address whether the spatial and geometric constraints of cells in the embryo restrict cellular heterogeneity in gene expression across space and time. These constraints are missing in adherent cultures where cells are free to move and able to make colonies of various sizes. This point can be addressed by seeding ESCs onto micropatterns of adhesive surfaces that constrain the movement of the cells and allow a more homogeneous, controlled differentiation in a high-throughput manner.

Subjecting micropatterned mouse and human PSCs to different signalling environments has reproduced early fate decisions including the emergence of germ layers and their subdivisions [10-13] and an organiser-like population [14, 28] that expresses the marker GSC and can induce the formation of secondary axial structures on xenotransplantation to a recipient embryo. In these experiments, cells develop as coherent groups in defined relative proportions, with few 
heterogeneities; often organised into concentric rings of different cell types. Micropatterned cultures are a useful tool to decipher the signalling logic associated with the early spatial organization of tissue primordia [15] and have highlighted the importance of signal dynamics [16$18]$, boundary conditions [11], mechanics, geometry $[13,19,20]$ and community $[21]$ in cell fate decisions. In an extension of this work, the radial symmetry observed with circular micropatterns could be broken by applying an asymmetric flow of signals and inhibitors in a situation that mimics the embryo [22].

In attempting to model gastrulation [12,13], micropatterned PSCs can undergo a regionalised EMT to form a multi-layered ring within the pattern $[10,13]$ that appears to move centrally over time [12]. While the local arrangement of cells in the T/BRA expressing region may be reminiscent of those at the primitive streak, the resulting mesodermal and endodermal subpopulations are locally clustered in a manner inconsistent with an overall body plan [12]. Adherent ESC and micropattern differentiation systems can help us to understand the local cell dynamics at gastrulation but they do not capture the overall migration and resolution of germ layer subpopulations or morphogenesis of the embryo. Micropatterns are an excellent model to explore the phase space of response of intrinsic Gene Regulatory Networks (GRNs) to signals (mechanical and chemical) but they are limited by their inability to evolve over time and their intrinsic tendency towards radial symmetry. This has been recently shown by changing the mechanical properties of the cellular substrate. Most micropatterned cultures are grown on Laminin-coated glass surfaces, but changing the cellular substrate to compliant polyacrylamide hydrogels generates local gastrulation-like nodes instead of a ring structure [23]. Compliant substrates permit local changes to cell density and adhesion, with the initiation of a gastrulation-like process at sites of highest cell adhesion tension. While signalling influences the proportions of different germ layers in this system, the local mechanical environment can determine the degree and localisation of gastrulation-like events. Overall, a greater understanding of the influence of mechanics and geometry on these GRNs will extend their utility as a model system.

\section{Blastoids and Peri-implantation Organoids - models of pre-gastrulation development}

While differentiation in adherent culture has revealed the roles of chemical, physical and geometrical constraints on early development, it does not reflect the three-dimensional organization of the embryo. Indeed, micropatterned cultures have raised the question of whether a non-adherent culture environment would provide further insights on cell fate decisions and 
patterning when these constraints are relaxed. Embryoid bodies, resulting from the differentiation of ESC aggregates in suspension have been used to explore this aspect of differentiation [24,25]. While EBs may show ordered phases of gene expression [26] and in some instances patterning asymmetries [27], these events are not accompanied by changes in gross morphology as in the embryo. Furthermore, subsequent differentiation is chaotic and produces spatially disordered groups of cell types. Nevertheless, these models have highlighted the importance of their dimensions as T/Bra can be seen to localise to one pole in a size-dependent manner [26,27]. An example that should not be overlooked is the formation of organiser-like cells in EBs formed from human ESCs that can induce the formation of a secondary body axis on transplantation to the frog embryo [28]. The observation of patterning asymmetries in three-dimensional ESC cultures is remarkable since signals from the extra-embryonic tissues are thought to direct symmetrybreaking in vivo.

The derivation of stem cells for the extra-embryonic lineages has enabled 3D in vitro modelling of peri-implantation development through self-organisation alongside ESCs. The trophectoderm has been captured in cell lines called Trophoblast Stem cells (TSCs [29]) and the primitive endoderm as Extra-Embryonic Endoderm cells (XEN, mouse [30]; nEnd, human [31]). When ESCs are combined with TS cells, they form structures reminiscent of early blastocysts, called blastoids. Within the blastoid environment, the TS cells convert into more TE-like cysts and ESCs progress to epiblastlike and small PrEnd subpopulations [32-34]. Blastoids form more efficiently when the TS-cell population has been pre-cultured in an environment favouring a pre-implantation polar trophoblast-like state [35].

When mouse ESCs are combined with TSCs within Matrige ${ }^{\circledR}$, they adopt an architecture and a bias of Brachyury expression in a manner reminiscent of the embryo [36]. However, they lack anterior fates such as neural ectoderm and the axial mesoderm of the head process, and they do not progress beyond a peri-gastrulation morphology (ie. a cup-shaped epithelium). By adding XEN cells to the TS/ESC combination, similar peri-gastrulation structures can develop in the absence of Matrige ${ }^{\circledR}$ and they are more complex, with specification of anterior fates such as anterior visceral endoderm-like cells in the XEN cell layer and posterior endoderm in a spatially organised way [37].

Collectively, these PSC co-culture systems recreate the dynamics of cell sorting in the first two fate decisions of mouse embryogenesis and later in the formation of the gastrulation-stage embryonic 
architecture. They reflect a more complex configuration of cells than in micropatterned cultures through the use of both embryonic and extra-embryonic cell types and their three-dimensional organisation. Cellular dynamics are similar in both formats, with the occurrence of movement, sorting and localised emergence of mesoderm reminiscent of gastrulation. Implanting blastoids into the uterus or extending the culture of ETX-embryos does not, however, enable further development [38]. This intriguing shortcoming invites investigation into the importance of uterine factors and the mechanical microenvironment in establishing and maintaining post-implantation development. Recent work with blastoid culture in chemically defined conditions suggests that the barriers preventing further blastoid development beyond implantation, might soon be overcome $[33,34,39]$. Notable examples include the use of extended pluripotent stem cells $[33,34]$ and chemically inductive culture conditions [39] to achieve a range of extra-embryonic cell types and an architecture that is consistent with the post-implantation embryo.

While much of this work has been conducted with mouse cells, a variety of organoid systems now exist that model the peri-implantation human embryo. Human ESCs cultured in hydrogels can actively break symmetry in a cell density-dependent manner to form 3D asymmetric cysts known as post-implantation amniotic sac embryoids (PASEs) [40]. They can develop a primitive streak-like phenotype in a manner that is regulated by BMP-SMAD signalling to produce structures similar to the day 10 human epiblast [41]. This approach has been further refined by incorporating the controlled environment of a microfluidic chip, enabling more robust symmetry breaking and the emergence of primitive streak derivatives such as primordial germ cells [42]. Advances in this field have revealed the capacity for stem cell cultures to recapitulate peri-implantation embryogenesis and enable in vitro investigation of this otherwise intractable stage of mammalian development.

\section{Gastruloids - models of body plan specification}

In 2009, Yusuke Marikawa reported that aggregates of small numbers of Embryonal Carcinoma cells self-organized polarized structures with mesodermal characteristics that are reminiscent of exogastrulae [43]. Extending these experiments to mouse ESCs, with an experimental design derived from differentiation in adherent culture [44] led to the development of gastruloids: freefloating aggregates of defined numbers of PSCs that undergo symmetry breaking, germ layer specification and axial organization ([453-48]). Gastruloids develop on a similar time course to the embryo until the equivalent of midgestation and generate derivatives of the mesoderm and endoderm. They are devoid of brain tissue but include cell fates associated with the spinal cord 
[47]. Spatial transcriptomics has shown that gene expression in gastruloids is very similar to that in mouse embryos, with the most marked difference being the lack of gene expression associated with anterior neuronal cell types [48]. Variation of the culture conditions around the equivalent of gastrulation stages promotes interactions between tissues and the development of a cardiac primordium [49].

Embedding gastruloids in diluted Matrige ${ }^{\circledR}$ promotes their segmentation to form patterned somite-like structures and an ordered posterior neural tube $[48,50]$. The initial arrangement of tissues is quite different to the gastrulation-stage embryo; early gastruloids are solid spheroids of ESCs rather than a cup-shaped epithelium like the epiblast and they are not covered by the extraembryonic tissues as in the embryo. Nevertheless, gastruloids develop a topology and gene expression profile consistent with the embryo at early organogenesis; especially when grown in a supportive extracellular matrix. Notably, they have been observed to form populations of primordial germ cell-like cells and tracts of gut endoderm $[47,48,50]$. Histological organisation likely occurs through large-scale cell migration and wholescale morphogenesis, taking mechanical cues from the extracellular matrix. Collectively, gastruloid cultures reveal an intrinsic capacity for mouse ESCs to self-organise when cultured in suspension at defined numbers in the absence of structured external signalling. The relationship between genetics and mechanics in this system remains to be investigated further and it will be particularly exciting to see how 3D cultures of human ESCs can be used to model features of early human development and teratology (see [51, 52]: HESCA-CSR and neuronal organoids).

Altogether, these cultures offer appropriate model systems to study the mechanisms mediating pattern formation and cellular interactions throughout development. These insights will be particularly important in the realm of human embryology, where both the availability of embryonic sample material and the capacity for experimentation are restricted. Adherent cultures of PSCs have informed us about the regulation and process of cell-fate specification, exploiting the highly controlled micropattern platform to tease apart the influences of geometry and confinement in this process. Blastoids and peri-implantation organoids have shown how the different compartments of the pre-gastrulation embryo interact with one another to establish its three-dimensional architecture and invite investigation into the role of uterine factors in regulating early development. Gastruloids add to this picture by revealing the capacity for the embryonic component to develop its own body plan, undergo morphogenesis and to establish the tissue interactions that allow for the segregation and subdivision of the germ layers. 
These approaches complement conventional embryology, but it is also important to be aware of their limitations to be able to learn from them. The constrained growth environment of micropatterned cell cultures may limit their potential to form complex tissues, but it is exactly this constraint that has made them so informative about how their patterning emerges. Gastrulationlike cell emergence is observed at a low frequency in ETX-embryos, but this may underline the importance of achieving a balanced presence and interaction of the extra-embryonic components in the conceptus. Gastruloids may be an abstracted system with a different morphology to the gastrula, but they reflect the result of development in an unconstrained environment and the intrinsic capacity for ESC self-organisation. We can learn from these constructed systems as long as they are robust and reproducible. These models demonstrate the ways in which cultures of pluripotent stem cells can be used to gain different perspectives on embryonic development in a way that greatly extends what we can learn from observations of the embryo alone.

\section{Author Contributions:}

Peter Baillie-Benson: Conceptualisation, Writing - Review \& Editing, Visualisation. Naomi Moris: Conceptualisation, Writing - Review \& Editing. Alfonso Martinez Arias: Conceptualisation, Writing - Original Draft, Writing - Review \& Editing, Funding Acquisition.

\section{Funding}

This work was supported by the European Research Council (Advanced Grant to A.M.A., number 834580) and the Leverhulme Trust (RPG-2018-356). N.M. holds the Constance Work Junior Research Fellowship at Newnham College, Cambridge.

\section{References:}

1. Anderson K V., Ingham PW: The transformation of the model organism: A decade of developmental genetics. Nat Genet 2003, 33:285-293.

2. Hamburger V: The Heritage of Experimental Embryology: Hans Spemann and the Organizer. Oxford University Press; 1988.

3. Rossant J, Tam PPL: Blastocyst lineage formation, early embryonic asymmetries and axis patterning in the mouse. Development 2009, 136:701-713.

4. Rivera-pérez JA, Hadjantonakis A, Wu DK, Kelley MW, Nichols J, Smith A: The Dynamics of Morphogenesis in the Early Mouse Embryo. 2014, doi:10.1101/cshperspect.a015867.

5. Tam PPL, Behringer RR: Mouse gastrulation: The formation of a mammalian body plan. Mech Dev 1997, 68:3-25.

6. McCauley HA, Wells JM: Pluripotent stem cell-derived organoids: Using principles of developmental biology to grow human tissues in a dish. Dev 2017, 144:958-962.

7. Lowell S, Benchoua A, Heavey B, Smith AG: Notch promotes neural lineage entry by pluripotent embryonic stem cells. PLoS Biol 2006, 4:805-818.

8. Turner D a, Trott J, Hayward P, Rué P, Martinez Arias A: An interplay between extracellular signalling and the dynamics of the exit from pluripotency drives cell fate decisions in mouse ES cells. Biol Open 2014, 3:614-26. 
9. Martinez Arias A, Brickman JM: Gene expression heterogeneities in embryonic stem cell populations: origin and function. Curr Opin Cell Biol 2011, 23:650-6.

10. Warmflash A, Sorre B, Etoc F, Siggia ED, Brivanlou AH: A method to recapitulate early embryonic spatial patterning in human embryonic stem cells. Nat Methods 2014, doi:10.1038/nmeth.3016.

11. Etoc F, Metzger J, Ruzo A, Kirst C, Yoney A, Ozair MZ, Brivanlou AH, Siggia ED: A Balance between Secreted Inhibitors and Edge Sensing Controls Gastruloid Self-Organization. Dev Cell 2016, 39:302-315.

12. Morgani SM, Metzger JJ, Nichols J, Siggia ED, Hadjantonakis A-K: Micropattern differentiation of mouse pluripotent stem cells recapitulates embryo regionalized fates and patterning. Elife 2017, doi:10.1101/236562.

13. Martyn I, Siggia ED, Brivanlou AH: Mapping cell migrations and fates in a gastruloid model to the human primitive streak. Dev 2019, 146.

14. - Martyn I, Kanno TT, Ruzo A, Siggia EE, Brivanlou AA: Self-organization of a functional human organizer by combined WNT and NODAL signalling. Nature 2018, doi:10.1101/234633.

The authors describe a population of organiser-like cells in micropatterned cultures of hESCs. On grafting this population to the chicken embryo, these cells form condensed axial mesodermal tissues and appear to induce structures reminiscent of secondary body axes.

15. Britton G, Heemskerk I, Hodge R, Qutub AA, Warmflash A: A novel self-organizing embryonic stem cell system reveals signaling logic underlying the patterning of human ectoderm. Dev 2019, 146.

16. Yoney A, Etoc F, Ruzo A, Carroll T, Metzger JJ, Martyn I, Li S, Kirst C, Siggia ED, Brivanlou AH: WNT signaling memory is required for ACTIVIN to function as a morphogen in human gastruloids. Elife 2018, 7:1-28.

17. $\bullet$ Chhabra S, Liu L, Goh R, Kong X, Warmflash A: Dissecting the dynamics of signaling events in the BMP, WNT, and NODAL cascade during self-organized fate patterning in human gastruloids. PLOS Biol 2019, 17:1-38.

Chhabra et al. present a quantitative study of the signalling dynamics responsible for generating pattern in micropatterned colonies of human ESCs. They find that there is no stable signalling gradient across the tissue and instead it is the duration of signalling and boundary effects that lead to pattern and challenge a causal role for Turing-like mechanisms in the pattern.

18. Heemskerk I, Burt K, Miller M, Chhabra S, Guerra MC, Liu L, Warmflash A: Rapid changes in morphogen concentration control self-organized patterning in human embryonic stem cells. Elife 2019, 8:1-28.

19. Blin G, Wisniewski D, Picart C, Thery M, Puceat M, Lowell S: Geometrical confinement controls the asymmetric patterning of Brachyury in cultures of pluripotent cells. Development 2018, doi:10.1242/dev.166025.

20. Xue X, Sun Y, Resto-Irizarry AM, Yuan Y, Aw Yong KM, Zheng Y, Weng S, Shao Y, Chai Y, Studer $L$, et al.: Mechanics-guided embryonic patterning of neuroectoderm tissue from human pluripotent stem cells. Nat Mater 2018, 17:633-641.

21. Nemashkalo A, Ruzo A, Heemskerk I, Warmflash A: Morphogen and community effects determine cell fates in response to BMP4 signaling in human embryonic stem cells. Dev 2017, 144:3042-3053.

22. - Manfrin A, Tabata Y, Paquet ER, Vuaridel AR, Rivest FR, Naef F, Lutolf MP: Engineered 
signaling centers for the spatially controlled patterning of human pluripotent stem cells. Nat Methods 2019, 16:640-648.

This work provides the first report of breaking the radial symmetry of micropatterned hPSC cultures by exposing them to controlled morphogen gradients in a microfluidic chip. By engineering signalling centres, they direct patterning and they characterise the effects of morphogen concentration and cell density on the cellular response.

23. $\bullet$ Muncie JM, Ayad NME, Lakins JN, Weaver VM: Mechanics regulate human embryonic stem cell self-organization to specify mesoderm. bioRxiv (preprint) 2020, doi:https://doi.org/10.1101/2020.02.10.943076.

By culturing hESCs on patterned soft substrates, Muncie et al. find that substrate compliance and geometry promote the formation of mesoderm through activation of $\mathrm{Wnt} / \beta$-catenin signalling. These findings highlight the interplay between geometry, mechanics and signalling in cell fate specification and encourage a re-assessment of the concentric mesodermal rings observed in micropatterned hESC cultures.

24. Brickman JM, Serup P: Properties of embryoid bodies. Wiley Interdiscip Rev Dev Biol 2017, 6:1-11.

25. Doetschman TC, Eistetter H, Katz M, Schmidt W, Kemler R: The in vitro development of blastocyst-derived embryonic stem cell lines: formation of visceral yolk sac, blood islands and myocardium. J Embryol Exp Morphol 1985, 87:27-45.

26. Leahy AMY, Xiong J, Kuhnert F, Stuhlmann H: Use of Developmental Marker Genes to Define Temporal and Spatial Patterns of Differentiation During Embryoid Body Formation. J Exp Zool 1999, 81:67-81.

27. ten Berge D, Koole W, Fuerer C, Fish M, Eroglu E, Nusse R: Wnt signaling mediates selforganization and axis formation in embryoid bodies. Cell Stem Cell 2008, 3:508-18.

28. Sharon N, Mor I, Golan-Lev T, Fainsod A, Benvenisty N: Molecular and functional characterizations of gastrula organizer cells derived from human embryonic stem cells. Stem Cells 2011, 29:600-608.

29. Tanaka S, Kunath T, Hadjantonakis AK, Nagy A, Rossant J: Promotion of trophoblast stem cell proliferation by FGF4. Science (80- ) 1998, 282:2072-2075.

30. Kunath T, Arnaud D, Uy GD, Okamoto I, Chureau C, Yamanaka Y, Heard E, Gardner RL, Avner $P$, Rossant $\mathrm{J}$ : Imprinted $\mathrm{X}$-inactivation in extra-embryonic endoderm cell lines from mouse blastocysts. Development 2005, 132:1649-1661.

31. Linneberg-Agerholm M, Wong YF, Herrera JAR, Monteiro RS, Anderson KGV, Brickman JM: Naïve human pluripotent stem cells respond to Wnt, Nodal and LIF signalling to produce expandable naïve extra-embryonic endoderm. Dev 2019, 146.

32. $\bullet$ Rivron NC, Frias-Aldeguer J, Vrij EJ, Boisset J-C, Korving J, Vivié J, Truckenmüller RK, van Oudenaarden A, van Blitterswijk $\mathrm{CA}^{\dagger}$, Geijsen $\mathrm{N}^{\dagger}$ : Blastocyst-like structures generated solely from stem cells. Nature 2018, doi:10.1038/s41586-018-0051-0.

This study gives the first description of blastocyst-like structures generated from the assembly of mouse embryonic and trophoblast stem cells in reliable numbers. The authors find that interactions between these components maintain trophoblast proliferation and self-renewal, particularly through BMP and Nodal signalling. On transferring these structures to recipient female mice, they implant and trigger deciduation but do not develop further.

33. Sozen B, Cox AL, De Jonghe J, Bao M, Hollfelder F, Glover DM, Zernicka-Goetz M: SelfOrganization of Mouse Stem Cells into an Extended Potential Blastoid. Dev Cell 2019, 
51:698-712.e8.

34. Li R, Zhong C, Yu Y, Liu H, Sakurai M, Yu L, Min Z, Shi L, Wei Y, Takahashi Y, et al.: Generation of Blastocyst-like Structures from Mouse Embryonic and Adult Cell Cultures. Cell 2019, 179:687-702.e18.

35. Frias-Aldeguer J, Kip M, Vivié J, Li L, Alemany A, Korving J, Darmis F, Oudenaarden A van, Blitterswijk CA Van, Geijsen N, et al.: Embryonic signals perpetuate polar-like trophoblast stem cells and pattern the blastocyst axis. bioRxiv (preprint) 2020, doi:10.1101/510362.

36. Harrison SE, Sozen B, Christodoulou N, Kyprianou C, Zernicka-goetz M: Assembly of embryonic and extra-embryonic stem cells to mimic embryogenesis in vitro. Science (80- ) 2017, doi:10.1126/science.aal1810.

37. - Sozen B, Amadei G, Cox A, Wang R, Na E, Czukiewska S, Chappell L, Voet T, Michel G, Jing $N$, et al.: Self-assembly of embryonic and two extra-embryonic stem cell types into gastrulating embryo-like structures. Nat Cell Biol 2018, 20:979-989.

Sozen et al. describe the assembly of mouse embryonic, trophoblast and extra-embryonic endoderm cells to form structures with a very similar architecture to the pre-gastrula without using an artificial extracellular matrix. They observe a degree of antero-posterior patterning and localised epithelial-to-mesenchymal transition at a low frequency.

38. Zhang S, Chen T, Chen N, Gao D, Shi B, Kong S, West RC, Yuan Y, Zhi M, Wei Q, et al.: Implantation initiation of self-assembled embryo-like structures generated using three types of mouse blastocyst-derived stem cells. Nat Commun 2019, 10.

39. Vrij EJ, Reimer YSS op, Aldeguer JF, Guerreiro IM, Kind J, Koo B-K, Blitterswijk CA Van, Rivron NC: Chemically-defined induction of a primitive endoderm and epiblast-like niche supports post-implantation progression from blastoids. bioRxiv (preprint) 2019, doi:10.1101/510396.

40. Shao Y, Taniguchi K, Townshend RF, Miki T, Gumucio DL, Fu J: A pluripotent stem cell-based model for post-implantation human amniotic sac development. Nat Commun 2017, 8:115.

41. - Simunovic M, Metzger JJ, Etoc F, Yoney A, Ruzo A, Martyn I, Croft G, You DS, Brivanlou AH, Siggia ED: A 3D model of a human epiblast reveals BMP4-driven symmetry breaking. Nat Cell Biol 2019, 21:900-910.

Simunovic et al. present a 3D hESC culture system that resembles the day 10 human epiblast and show that antero-posterior symmetry-breaking is dependent on exposure to BMP and occurs in the absence of any extra-embryonic tissues. They show that WNT and DKK1 act downstream of BMP to regulate this process and suggest that both activator and inhibitor are expressed in the posterior epiblast.

42. $\bullet$ Zheng Y, Xue X, Shao Y, Wang S, Esfahani SN, Li Z, Muncie JM, Lakins JN, Weaver VM, Gumucio DL, et al.: Controlled modelling of human epiblast and amnion development using stem cells. Nature 2019, 573:421-425.

The authors present a considerable advance on their preceding work with hESC by forming and patterning epiblast-like structures within a microfluidic chip. They observe epiblast lumenogenesis, the formation of an amnion and a primitive streak-like cells as well as the differentiation to primordial germ cells. This system is particularly notable for being a robust and reproducible platform that exploits the controllability of microfluidics to produce these tissues at scale. 
43. Marikawa Y, Tamashiro DA a, Fujita TC, Alarcón VB: Aggregated P19 mouse embryonal carcinoma cells as a simple in vitro model to study the molecular regulations of mesoderm formation and axial elongation morphogenesis. Genesis 2009, 47:93-106.

44. Turner DA, Hayward PC, Baillie-Johnson P, Rue P, Broome R, Faunes F, Martinez Arias A: Wnt/ $\beta$-catenin and FGF signalling direct the specification and maintenance of a neuromesodermal axial progenitor in ensembles of mouse embryonic stem cells. Development 2014, 141:4243-4253.

45. van den Brink SC, Baillie-Johnson P, Balayo T, Hadjantonakis A-K, Nowotschin S, Turner DA, Martinez-Arias A: Symmetry breaking, germ layer specification and axial organisation in aggregates of mouse embryonic stem cells. Development 2014, 141:4231-4242.

46. Turner DA, Girgin M, Alonso-Crisostomo L, Trivedi V, Baillie-Johnson P, Glodowski CR, Hayward PC, Collignon J, Gustavsen C, Serup P, et al.: Anteroposterior polarity and elongation in the absence of extraembryonic tissues and spatially localised signalling in Gastruloids, mammalian embryonic organoids. Development 2017, doi:10.1242/dev.150391.

47. $\bullet$ Beccari L, Moris N, Girgin M, Turner DA, Baillie-Johnson P, Cossy A-C, Lutolf MP, Duboule $D$, Arias AM: Multi-axial self-organization properties of mouse embryonic stem cells into gastruloids. Nature 2018, doi:10.1038/s41586-018-0578-0.

This study describes axial patterning in gastruloids and finds that gene expression is structured along the three major body axes, including the spatial and temporal co-linear expression of the Hox gene clusters. In comparing the transcriptomes of gastruloids and embryos, we find a high degree of similarity in spatial and temporal patterns of gene expression to post-occipital embryonic development.

48. $\bullet \bullet$ van den Brink SC, Alemany A, van Batenburg V, Moris N, Blotenburg M, Vivié J, BaillieJohnson P, Nichols J, Sonnen KF, Martinez Arias A, et al.: Single-cell and spatial transcriptomics reveal somitogenesis in gastruloids. Nature 2020, doi:10.1038/s41586020-2024-3.

This study presents the first spatial transcriptomic study of gastruloids and finds that they show ordered patterns of gene expression that correspond closely to somitogenesis in vivo. We use the transcriptomic data to identify previously undescribed cell types in the gastruloids, including endothelial and haemato-endothelial cells, primordial germ cells and extra-embryonic ectoderm.

49. - Rossi G, Boni A, Guiet R, Girgin M, Kelly RG, Lutolf MP: Embryonic organoids recapitulate early heart organogenesis. bioRxiv (preprint) 2019, doi:10.1101/802181.

Rossi et al. present an in vitro model for early heart organogenesis in axially-organised mouse embryonic organoids. Importantly, their observations show that the tissue-tissue interactions between the first and second heart fields and with the endoderm can be recapitulated in an organoid system, in a spatially organised way.

50. $\bullet \bullet$ Veenvliet J V, Bolondi A, Kretzmer H, Haut L, Scholze-wittler M: Mouse embryonic stem cells self-organize into trunk-like structures with neural tube and somites. bioRxiv 2020, By embedding gastruloids in a low percentage of Matrigel, the authors demonstrate that an appropriate extracellular matrix can promote the formation of somite-like structures and an ordered neural tube in these cultures by inducing changes in ECM components. They also 
find that the somite-like compartments include dorsal and ventral subpopulations, indicating that gastruloids form a diverse range of embryonic progenitors. The study also recapitulate the $\mathrm{Tb} \mathrm{6}^{-1-}$ mutant phenotype.

51. Marikawa Y, Chen HR, Menor M, Deng Y, Alarcon VB: Exposure-based assessment of chemical teratogenicity using morphogenetic aggregates of human embryonic stem cells. Reprod Toxicol 2020, 91:74-91.

52. - Libby, A.R.G., Joy, D.A., Elder, N.H., Bulger, E.A., Krakora, M.Z., Gaylord, E.A., MendozaCamacho, F., McDevitt TC: Axial Elongation of Caudalized Human Pluripotent Stem Cell Organoids Mimics Neural Tube Development. bioRxiv (preprint) 2020.

In this work the authors show that under specific culture conditions, aggregates of human induced pluripotent stem cells can produce elongated structures with features of the spinal cord.

\section{Note for Figure 1:}

Developmental timelines were approximately aligned with reference to Dr Mark Hill's online Embryology resource (UNSW, Australia; last retrieved from https://embryology.med.unsw.edu.au/embryology/index.php/Main Page, April 8th 2020). Mouse embryo schematics were drawn with reference to the Medical Research Council's EMAP Anatomy Atlas (last retrieved from http://www.emouseatlas.org/emap/ema/home.php, April 8th 2020) and Dr Hill's Embryology resource. Human embryo schematics were prepared with reference to Dr Hill's Embryology resource and an online course in embryology produced by the Universities of Fribourg, Lausanne and Bern (last retrieved from http://www.embryology.ch/anglais/iperiodembry/carnegie01.html, April 8th 2020). The schematics for the model systems were prepared with reference to figures from: [32,33], mouse blastoids; [10,12], micropatterns; [37] mouse ETX-Embryos; [48,50], Gastruloids \& TLS; [41,42], human epiblast-like cysts; [51] HESCA-CSR.

[BOX 1] A Note on Nomenclature: The term organoid is generic and refers to stem cell-derived structures that resemble those in the organism. The term has led to naming particular structures with the suffix -oid. In the case of embryonic structures, several terms (blastoids, EPS-blastoids, ETS-embryos, ETX-embryos etc.) are used to reflect the similarity to embryonic structures and the difference with embryoid bodies. The term gastruloid was initially coined for three-dimensional free-floating structures that progress through a gastrulation-like process and have an axial extension. However, it is often also used in the context of two-dimensional micropatterned PSC cultures. We would suggest that, to avoid confusion, the structures derived from micropatterns are termed 2D-gastruloids or 'germ layer organoids' to reflect the difference with gastruloids. 


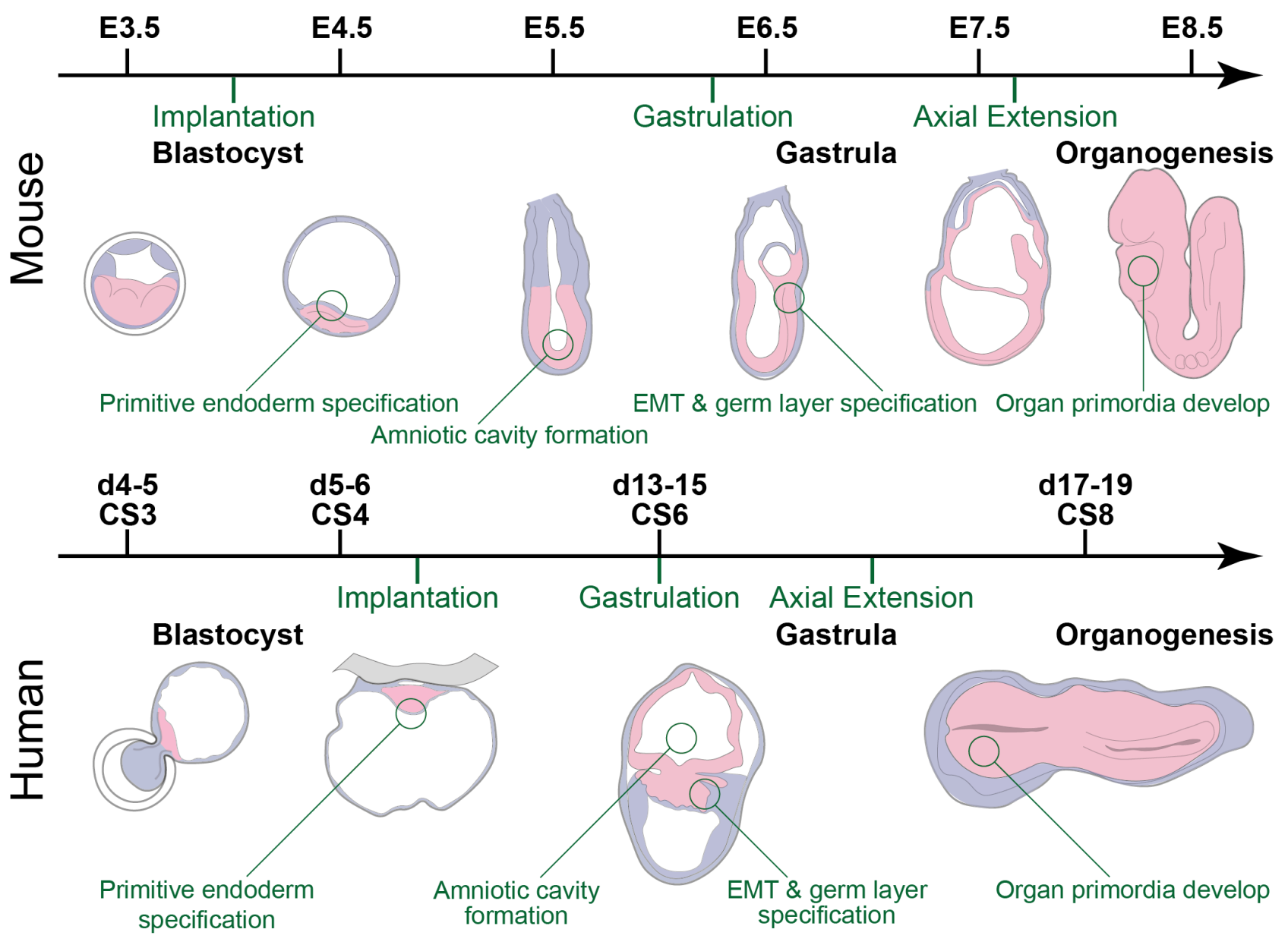

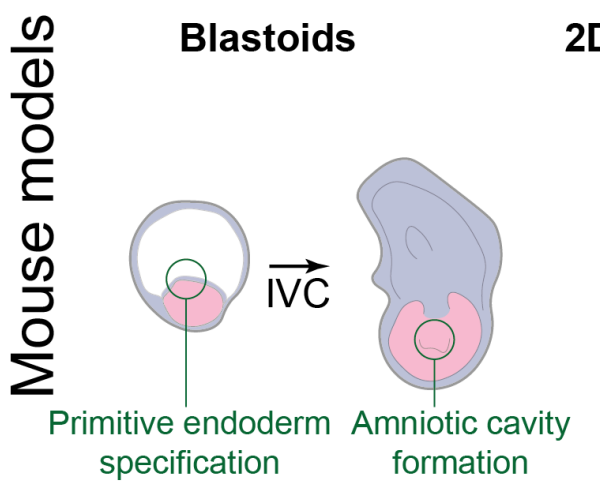

$\frac{0}{0}$
$\frac{0}{0}$
$\frac{0}{E}$
$\frac{1}{0}$
$\frac{1}{5}$
$\frac{1}{1}$

\section{D Micropatterns}

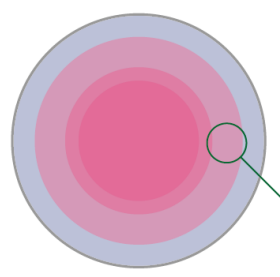

2D Micropatterns

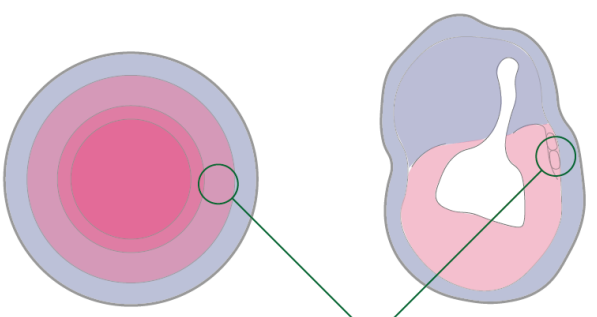

EMT \& germ layer specification
ETX-Embryos

Gastruloids \& TLS

Figure 1: An Overview of Mouse and Human Embryonic Development and Corresponding In Vitro Models.

Schematic representations of the developing mouse and human embryos are shown on the top two rows. The bottom two rows represent corresponding in vitro systems for modelling equivalent stages of embryonic development from cultures of mouse and human pluripotent stem cells. 
Embryonic components are coloured in pink throughout; extra-embryonic components in blue. Key developmental stages and processes are indicated with green text. The schematics are simple representations and are not drawn to scale. The Carnegie Stage (CS) 6 human embryo schematic is drawn from a posterior view and CS8 from a dorsal view; all other schematics represent transverse sections with anterior on the left (where appropriate). See Note For Figure 1 for references and abbreviations. 